\title{
Analytical Generalized Results for Handoff Probability in Wireless Networks
}

\author{
Yuguang Fang, Senior Member, IEEE, and Imrich Chlamtac, Fellow, IEEE
}

\begin{abstract}
In this letter we present analytical results for handoff probability for wireless networks under assumption that the call holding time and the cell residence time are all generally distributed. Easily computable formulas can be obtained for cases when the call holding time and cell residence time have rational Laplace transforms.
\end{abstract}

Index Terms-Call holding time, cell residence time, handoff probability, wireless networks.

\section{INTRODUCTION}

$\mathbf{T}$ ELETRAFFIC analysis for wireless networks, in particular, for Personal Communications Services (PCS), plays very important role in the network dimensioning and resource provisioning (see [4], [5], [10], and references therein). For these systems, contrary to the classical modeling assumptions of telephony systems, field trials show that call holding time, cell residence time and channel holding time are no longer exponentially distributed [1], [7], [10]. We observe that the channel holding time depends on the users' mobility, which in turn can be characterized by the cell residence time (dwell time), the time that a mobile user stays in a cell. Different services provided by the wireless networks are changing the calling habits of users, hence the call holding time (i.e., the time for a user connection requested by the user) is no longer exponentially distributed. In order to appropriately characterize performance metrics such as handoff probability, it is therefore necessary to have an appropriate distribution model for the cell residence time and call holding time to reflect the mobility of the users and the calling habits of users in a way consistent with field data. The distribution models with rational Laplace transforms, such as hyper-Erlang distribution [4] and the SOHYP distribution [10], can be used due to their generality of fitting field data. Moreover, such distribution models preserve the Markovian property of queueing network models desirable for obtaining analytical results for call blocking performance [4].

In this paper, we use general distribution models for the mobility and call holding time in order to obtain analytical characterization of several performance parameters. We concentrate on the handoff probability in this paper. The handoff probability is the probability that a call in a cell needs at least another handoff before its completion. It is an important quantity useful for the design of predictive handoff schemes [2], [3] and for the com-

Paper approved by N. C. Beaulieu, the Editor for Wireless Communication Theory of the IEEE Communications Society. Manuscript received July 24, 2000; revised July 12, 2001 and August 23, 2001. This work was supported in part by the National Science Foundation Faculty Early Career Development Award ANI0093241.

Y. Fang is with the Department of Electrical and Computer Engineering, University of Florida, Gainesville, FL 32611 USA (e-mail: fang@ece.ufl.edu).

I. Chlamtac is with the Erik Jonsson School of Engineering and Computer Science, University of Texas at Dallas, Richardson, TX 75083 USA (e-mail: chlamtac@utdallas.edu).

Publisher Item Identifier S 0090-6778(02)02028-7. putation of the handoff rate and handoff traffic rate [5], [11]. We derive formulas for handoff probability for the cases where the call holding time and cell residence time are distributed with general distributions. We then derive easily computable results for the cases where the call holding time and cell residence time have rational Laplace transforms.

\section{HANDOFF PROBABILITY}

Handoff probability is defined as the probability that a call needs at least one more handoff during its remaining life time. It characterizes whether an on-going call completes its session in the current cell or not. Obviously, this probability is useful in dimensioning resources in the neighboring cell. Depending on whether a call is a new call or a handoff call, we call the probability the handoff probability for a new call (NHOP) or the handoff probability for a handoff call (HHOP).

Let the call holding time $t_{c}$ (i.e., the unencumbered call holding time of requested connection to a wireless network for a new call, as in wireline telephony) be generally distributed with mean $1 / \mu$. Let $t_{m}$ be the cell residence time in the $m$ th cell a user transverses during its call life, $r_{1}$ be the time between the instant a new call is initiated and the instant the new call moves out of the cell if the new call is not completed, and let $r_{m}(m>1)$ be the residual life time distribution of call holding time when the call finishes the $m$ th handoff successfully. Let $t_{c}, t_{m}$, and $r_{1}$ have density functions $f_{c}(t), f(t)$, and $f_{r}(t)$ with their corresponding Laplace transforms $f_{c}^{*}(s), f^{*}(s)$, and $f_{r}^{*}(s)$.

We first study the handoff probability for a new call. Let $\sigma_{c}$ denote the set of poles of $f_{c}^{*}(-s)$ in the right half of the complex plane. Let $P_{n}$ denote the handoff probability for a new call (NHOP). Since a new call needs at least another handoff if and only if the call holding time $t_{c}$ is larger than the residual cell residence time $r_{1}$, hence we have (using inverse Laplace transform and the Residue Theorem [8])

$$
\begin{aligned}
P_{n} & =\operatorname{Pr}\left(r_{1} \leq t_{c}\right)=\int_{0}^{\infty} f_{r}(x) \int_{x}^{\infty} f_{c}(y) d y d x \\
& =\frac{1}{2 \pi j} \int_{\sigma-j \infty}^{\sigma+j \infty} \int_{0}^{\infty} f_{r}^{*}(s) e^{s x}\left[\int_{x}^{\infty} f_{c}(y) d y\right] d x d s \\
& =\frac{1}{2 \pi j} \int_{\sigma-j \infty}^{\sigma+j \infty} f_{r}^{*}(s) \int_{0}^{\infty}\left[\int_{x}^{\infty} f_{c}(y) d y\right] e^{s x} d x d s \\
& =\frac{1}{2 \pi j} \int_{\sigma-j \infty}^{\sigma+j \infty} f_{r}^{*}(s) \frac{-1+f_{c}^{*}(-s)}{s} d s \\
& =\frac{1}{2 \pi j} \int_{\sigma-j \infty}^{\sigma+j \infty} \frac{f_{r}^{*}(s)}{s} f_{c}^{*}(-s) d s \\
& =-\sum_{p \in \sigma_{c}} \operatorname{Res}_{s=p} \frac{f_{r}^{*}(s)}{s} f_{c}^{*}(-s)
\end{aligned}
$$

where $\sigma>0$ is a sufficiently small positive number. 


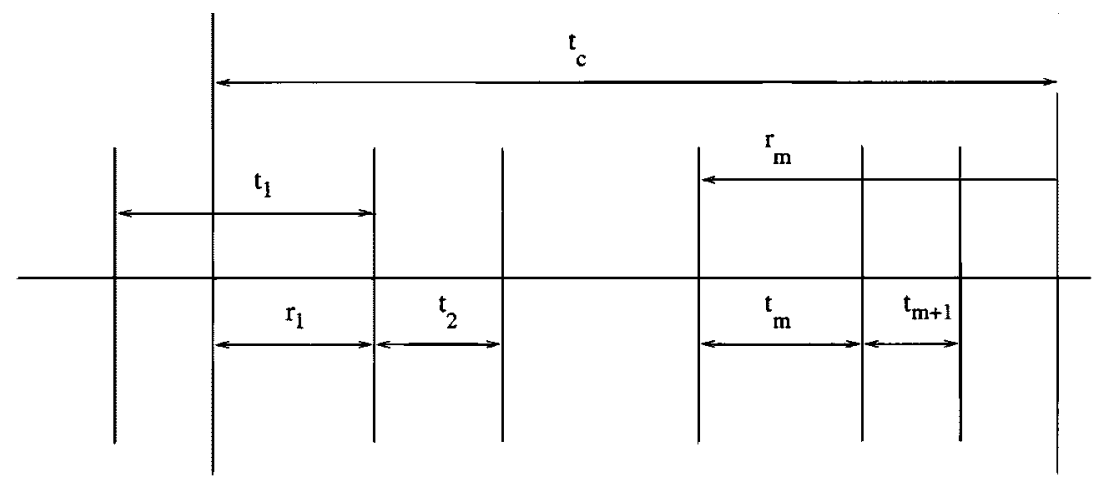

Fig. 1. The time diagram for call holding time and cell residence time.

Next we derive the handoff call handoff probability. This quantity is important as it allows us to monitor a call in progress and plan ahead for the next handoff of the call. Let $P_{h}(k)$ denote the probability that a handoff call after $(k-1)$ th handoff needs at least one more handoff in its remaining life time, hence $k \geq 2$. From the time diagram in Fig. 1, we obtain

$$
\begin{aligned}
P_{h}(k)=\operatorname{Pr}\left(r_{1}+t_{2}+\cdots\right. & +t_{k}+t_{k+1} \\
& \left.\leq t_{c} \mid r_{1}+t_{2}+\cdots+t_{k} \leq t_{c}\right) .
\end{aligned}
$$

We first compute the probability $\operatorname{Pr}\left(r_{1}+t_{2}+\cdots+t_{k}+t_{k+1} \leq\right.$ $t_{c}$ ). Let $\xi=r_{1}+t_{2}+\cdots+t_{k}+t_{k+1}$. Let $f_{\xi}(t)$ and $f_{\xi}^{*}(s)$ be the density function and the Laplace transform of $\xi$. From the independency of $r_{1}, t_{2}, t_{3}, \ldots$, we have

$$
f_{\xi}^{*}(s)=E\left[e^{-s \xi}\right]=E\left[e^{-s r_{1}}\right] \prod_{i=2}^{k+1} E\left[e^{-s t_{i}}\right]=f_{r}^{*}(s)\left(f^{*}(s)\right)^{k} .
$$

So the density function is given by

$$
f_{\xi}(t)=\frac{1}{2 \pi j} \int_{\sigma-j \infty}^{\sigma+j \infty} f_{r}^{*}(s)\left(f^{*}(s)\right)^{k} e^{s t} d s .
$$

Also, the Laplace transform of $\operatorname{Pr}(\xi \leq t)$ is $f_{\xi}^{*}(s) / s$. From (1) and the Residue Theorem, we obtain

$$
\begin{aligned}
\operatorname{Pr} & \left.r_{1}+t_{2}+\cdots+t_{k+1} \leq t_{c}\right) \\
& =\int_{0}^{\infty} \operatorname{Pr}(\xi \leq t) f_{c}(t) d t \\
& =\int_{0}^{\infty} \frac{1}{2 \pi j} \int_{\sigma-j \infty}^{\sigma+j \infty} \frac{f_{r}^{*}(s)\left[f^{*}(s)\right]^{k}}{s} e^{s t} d s f_{c}(t) d t \\
& =\frac{1}{2 \pi j} \int_{\sigma-j \infty}^{\sigma+j \infty} \frac{f_{r}^{*}(s)\left[f^{*}(s)\right]^{k}}{s} f_{c}^{*}(-s) d s \\
& =-\sum_{p \in \sigma_{c}} \operatorname{Res}_{s=p} \frac{f_{r}^{*}(s)\left[f^{*}(s)\right]^{k}}{s} f_{c}^{*}(-s) d s .
\end{aligned}
$$

From this and (1), applying the conditional probability argument, we obtain

$$
\begin{aligned}
P_{h}(k) & =\frac{\operatorname{Pr}\left(r_{1}+t_{2}+\cdots+t_{k}+t_{k+1} \leq t_{c}, r_{1}+t_{2}+\cdots+t_{k} \leq t_{c}\right)}{\operatorname{Pr}\left(r_{1}+t_{2}+\cdots+t_{k} \leq t_{c}\right)} \\
& =\frac{\operatorname{Pr}\left(r_{1}+t_{2}+\cdots+t_{k}+t_{k+1} \leq t_{c}\right)}{\operatorname{Pr}\left(r_{1}+t_{2}+\cdots+t_{k} \leq t_{c}\right)}
\end{aligned}
$$

$$
=\frac{\sum_{p \in \sigma_{c}} \operatorname{Res}_{s=p} \frac{f_{r}^{*}(s)\left[f^{*}(s)\right]^{k}}{s} f_{c}^{*}(-s)}{\sum_{p \in \sigma_{c}} \operatorname{Res}_{s=p} \frac{f_{r}^{*}(s)\left[f^{*}(s)\right]^{k}}{s} f_{c}^{*}(-s)} .
$$

In summary, we obtain the following theorem.

Theorem: Assume that the call holding time and cell residence time are generally distributed with Laplace transforms $f^{*}(s)$ and $f_{c}^{*}(s)$. Suppose that $f_{c}^{*}(s)$ has only finite number of isolated singular points in the left half complex plane (which is the case when it is rational function), then the handoff probabilities are given by

$$
\begin{gathered}
P_{n}=-\sum_{p \in \sigma_{c}} \operatorname{Res}_{s=p} \frac{f_{r}^{*}(s)}{s} f_{c}^{*}(-s), \\
P_{h}(k)=\frac{\sum_{p \in \sigma_{c}} \operatorname{Res}_{s=p} \frac{f_{r}^{*}(s)\left[f^{*}(s)\right]^{k}}{s} f_{c}^{*}(-s)}{\sum_{p \in \sigma_{c}} \operatorname{Res}_{s=p} \frac{f_{r}^{*}(s)\left[f^{*}(s)\right]^{k-1}}{s} f_{c}^{*}(-s)}
\end{gathered}
$$

where $\sigma_{c}$ is the set of poles of $f_{c}^{*}(-s)$ in the right half complex plane. In particular, when the call holding time is exponentially distributed with parameter $\mu$, we have

$$
P_{n}=f_{r}^{*}(\mu), \quad P_{h}(k)=f^{*}(\mu) .
$$

When the call holding time is Erlang distributed with parameter $(m, \mu)$, which has the following probability density function and Laplace transform:

$f_{c}(t)=\frac{(m \mu)^{m} t^{m-1}}{(m-1) !} e^{-m \mu t}, \quad f_{c}^{*}(s)=\left(\frac{m \mu}{s+m \mu}\right)^{m}$

then, we have $\left(g^{(p)}(x)\right.$ denotes the $i$ th derivative of function $g(x)$ at point $x)$

$$
\begin{aligned}
P_{n}= & \sum_{i=0}^{m-1} \frac{f_{r}^{*(i)}(m \mu)}{i !}(-m \mu)^{i}, \\
P_{h}(k)= & \frac{\sum_{i=0}^{m-1} \frac{\left.\left\{f_{r}^{*}(s)\left[f^{*}(s)\right]^{k}\right\}^{(i)}\right|_{s=m \mu}}{i !}(-m \mu)^{i}}{\sum_{i=0}^{m-1} \frac{\left.\left\{f_{r}^{*}(s)\left[f^{*}(s)\right]^{k-1}\right\}^{(i)}\right|_{s=m \mu}}{i !}(-m \mu)^{i}} .
\end{aligned}
$$




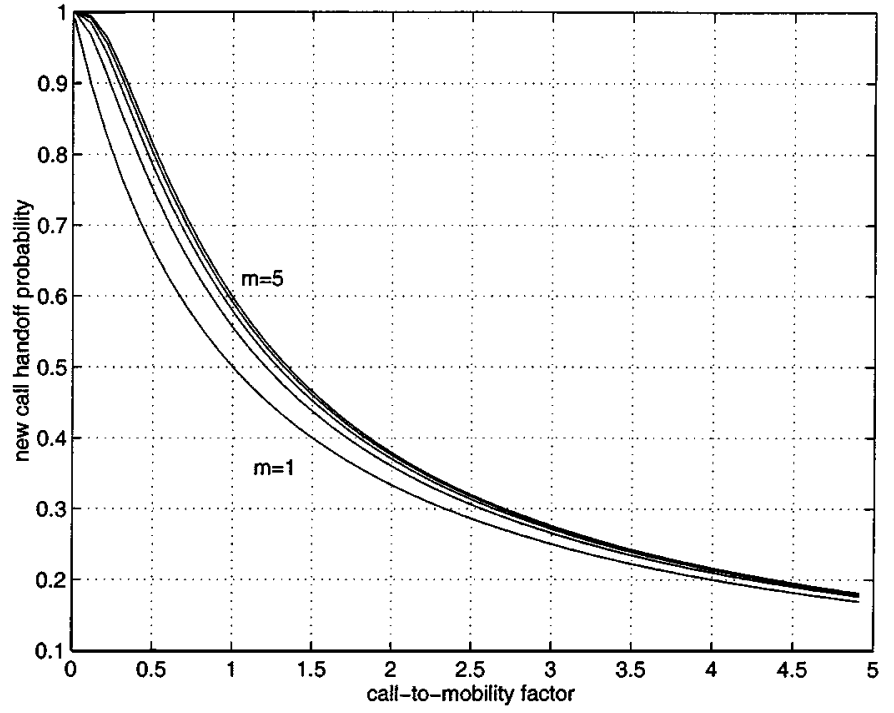

Fig. 2. New call handoff probability versus call-to-mobility factor.

When call holding time is not exponentially distributed, the handoff probability for a handoff call has not even been defined in the past. When the call holding time is exponentially distributed while the cell residence time is distributed with certain distributions (such as generalized Gamma distributed), similar results for handoff probability have been obtained [2], [9], [11]. It is important to observe that as long as the Laplace transform of the call holding time is rational, this theorem can be easily used to compute the handoff probability. Explicit results can be obtained for the case when the call holding time is hyper-Erlang distributed, the details are omitted due to the space limitation.

Next, we briefly study how the mobility and traffic parameters affect the handoff probability. When the call holding time is exponentially distributed with parameter $\mu$, and the cell residence time is also exponentially distributed with parameter $\eta$, we have $P_{n}=P_{h}(k)=1 /(1+\rho)$, where $\rho=\mu / \eta$ is called the call-to-mobility factor. It can be observed that as $\rho$ decreases, the handoff probability increases.

Assume now that the cell residence time is exponentially distributed with parameter $\eta$ and the call holding time is Erlang distributed with parameter $(m, \mu)$. From the residual life theorem, we know that the residual cell residence time $r_{1}$ is also exponentially distributed with $f_{r}^{*}(s)=f^{*}(s)=\eta /(s+\eta)$. From the Theorem, we obtain $P_{n}=1-[1-1 /(1+m \rho)]^{m}$. The handoff call handoff probability is given by

$$
\begin{aligned}
P_{h}(k)= & \frac{\sum_{i=0}^{m-1}\left(\begin{array}{c}
k+i-1 \\
i
\end{array}\right)\left(\frac{\alpha}{\alpha+\eta}\right)^{i}\left(\frac{\eta}{\alpha+\eta}\right)^{k}}{\sum_{i=0}^{m-1}\left(\begin{array}{c}
k+i-2 \\
i
\end{array}\right)\left(\frac{\alpha}{\alpha+\eta}\right)^{i}\left(\frac{\eta}{\alpha+\eta}\right)^{k-1}} \\
= & \frac{\sum_{i=0}^{m-1}\left(\begin{array}{c}
k+i-1 \\
i
\end{array}\right)\left(\frac{\eta}{\alpha+\eta}\right)^{i}\left(\frac{\eta}{\alpha+\eta}\right)^{k}}{\sum_{i=0}^{m-1}\left(\begin{array}{c}
k+i-2 \\
i
\end{array}\right)\left(\frac{\eta}{\alpha+\eta}\right)^{i}\left(\frac{\eta}{\alpha+\eta}\right)^{k-1}} .
\end{aligned}
$$
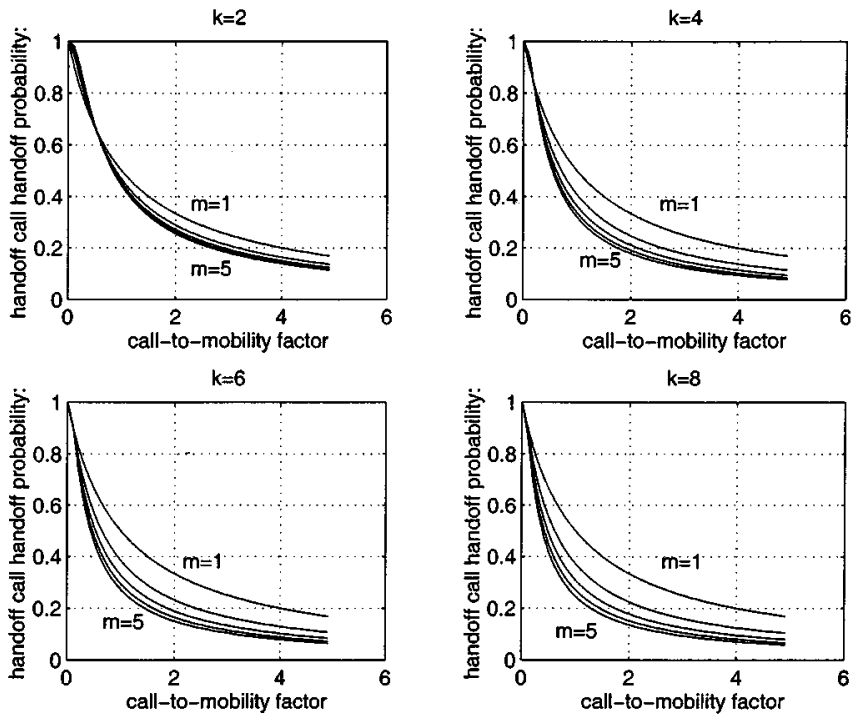

$k=8$

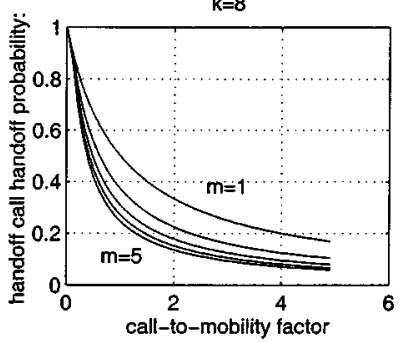

Fig. 3. Handoff call handoff probability versus call-to-mobility.

Fig. 2 shows the handoff probability for new calls vs. call-tomobility factor $(\rho)$. It shows that there is a significant difference between the handoff probabilities for the case when the call holding time is exponentially distributed and for the case when the call holding time is Erlang distributed, in particular when the mobility is higher (corresponding to the lower $\rho$ ). It is also shown that handoff probability is decreasing as the mobility is lower (i.e., when the call-to-mobility factor is higher). Fig. 3 shows the handoff call handoff probability. As for the new call handoff probability, the handoff call handoff probability is also decreasing as the call-to-mobility factor is increasing. As the number of handoffs increases, the difference between the cases for exponential call holding time and Erlang call holding time becomes more significant.

\section{CONCLUSION}

Handoff probability for wireless networks is an important parameter for predictive handoff design and general handoff traffic study. In this paper, we introduced generalized analytical results for handoff probability, to reflect the realistic cases of emerging wireless systems in which the call holding time is not exponentially distributed. Analytical results for handoff probability were derived under general call holding time and cell residence time.

\section{REFERENCES}

[1] F. Barcelo and J. Jordan, "Channel holding time distribution in cellular telephony," in Proc. 9th Int. Conf. on Wireless Communications, vol. 1, Calgary, AB, Canada, July, 9-11 1997, pp. 125-134.

[2] E. Del Re, R. Fantacci, and G. Giambene, "Handover and dynamic channel allocation techniques in mobile cellular networks," IEEE Trans. Veh. Technol., vol. 44, pp. 229-237, May 1995.

[3] — - "Efficient dynamic channel allocation techniques with handover queueing for mobile satellite networks," IEEE J. Select. Areas Commun., vol. 13, pp. 397-405, Feb. 1995.

[4] Y. Fang and I. Chlamtac, "Teletraffic analysis and mobility modeling for PCS networks," IEEE Trans. Commun., vol. 47, pp. 1062-1072, July 1999.

[5] Y. Fang, I. Chlamtac, and Y. B. Lin, "Channel occupancy times and handoff rate for mobile computing and PCS networks," IEEE Trans. Comput., vol. 47, pp. 679-692, June 1998. 
[6] D. Hong and S. S. Rappaport, "Traffic model and performance analysis for cellular mobile radio telephone systems with prioritized and nonprioritized handoff procedures," IEEE Trans. Veh. Technol., vol. 35, pp. 77-92, Aug. 1986.

[7] J. Jordan and F. Barcelo, "Statistical modeling of channel occupancy in trunked PAMR systems," in Proc. 15th Int. Teletraffic Conf. (ITC'15), V. Ramaswami and P. E. Wirth, Eds., 1997, pp. 1169-1178.

[8] W. R. LePage, Complex Variables and the Laplace Transform for Engineers. New York: Dover, 1980

[9] Y. B. Lin, S. Mohan, and A. Noerpel, "Queueing priority channel assignment strategies for handoff and initial access for a PCS network," IEEE Trans. Veh. Technol., vol. 43, pp. 704-712, Aug. 1994.
[10] P. Orlik and S. S. Rappaport, "A model for teletraffic performance and channel holding time characterization in wireless cellular communication with general session and dwell time distributions," IEEE J. Select. Areas Commun., vol. 16, pp. 788-803, month? 1998.

[11] M. M. Zonoozi and P. Dassanayake, "User mobility modeling and characterization of mobility patterns," IEEE J. Select. Areas Commun., vol. 15, pp. 1239-1252, Oct. 1997. 